\title{
Numerical Study on CX-Neutral Transport and Wall-Sputtering in W7-AS Diverted Plasmas
}

 \\ a Max-Planck-Institut für Plasmaphysik, Teilinstitut Greifswald, Euratom Association, \\ Greifswald/Garching, Germany \\ ${ }^{\mathrm{b}}$ Institut für Plasmaphysik, Forschungszentrum Jülich Gmbh, Euratom Association, Trilateral Euregio \\ Cluster, D-52425 Jülich, Germany
}

\begin{abstract}
Using the 3D SOL transport code EMC3/EIRENE, the paper presents a parameter study on CX-neutral transport and Fe-release from the wall due to physical sputtering in W7-AS diverted plasmas. The studies are restricted to the CX-neutrals induced by divertor and wall recycling processes. SOL plasma and divertor and wall recycling process are self-consistently calculated by the EMC3/EIRENE code. Island density is scanned across different confinement regimes. Cross-field transport coefficients are varied to cover an appropriate uncertainty range. Core plasma is prescribed, with the density profile, however, being switched between a peaked and a flat form typically measured for low and high density diverted plasmas. Studied are the sensitivities of the flux, energy spectrum and origin of the CX-neutrals to these parameters. In particular, it is aimed to identify under what condition and to what extent the edge islands can protect the wall from physical sputtering of the CX-neutrals.
\end{abstract}

PACS: 52.25.Fi, 52.25.Vy, 52.40.Hf, 52.65.-y, 52.65.Pp

JNM keywords: Theory and Modeling, Sputtering, Radiation Sources, Plasma-Materials Interaction

PSI-17 keywords: W7-AS, Island divertor, Impurity sources, Recycling, Edge modeling

*Corresponding author address: Wendelsteinstr. 1, 17491 Greifswald, Germany

*Corresponding author E-mail: feng@ipp.mpg.de 


\section{Introduction}

Plasma density in a current-free stellarator like W7-AS is usually limited by impurity radiation [1]. CX-neutrals induced by recycling process or by auxiliary particle fueling like gas-puff and beam-injection are considered to be a potential source of impurity production on plasma-facing components by means of sputtering. In fact, reducing the impurity sputtering yield, ionizing the impurities in the SOL and sweeping them back to the target are basic design objectives of a divertor. Previous EMC3/EIRENE studies on the target-released carbon transport in the W7-AS island divertor have shown that, by enhancing the recycling flux and lowering the island temperature, the impurity flow velocity, which is determined by the balance between thermal force and friction, can be switched from inwards- to outwards-directed [2]. A frictional plasma flow under enhanced recycling conditions can efficiently flush the carbon back to the targets and thereby strongly reduce the carbon concentration around the inner separatrix. On the other hand, however, the total carbon yield increases with increasing the recycling flux because of chemical effects, which, to a large extent, compensates the transport flushing effect. Nevertheless, light impurities like carbon do not significantly contribute to core radiation. The focus of this paper is turned to the wall-released iron which is observed to be one of main radiation impurity species in the core [3]. We pay our main attention to the transport of the recycling-induced CX-neutrals and investigate the role of the island divertor in protecting the wall from neutral sputtering. The iron production rates resulting from physical sputtering process, which are self-consistently calculated by the EIRENE code, serve as a quantity for measuring the sputtering-relevant neutral flux, rather than for providing a reliable, absolute Fe-release because of the existing uncertainties in $\mathrm{T}_{\mathrm{i}^{-}}$ profile and in wall-conditioning.

\section{Computational set-up}

In order to take the charge-exchange process in the core into account, the computation domain for neutrals is extended from the wall to the plasma center, as sketched in Fig. 1. The common SOL region for EMC3 [4] and EIRENE [5] starts from a closed flux surface just inside the inner separatrix and extends radially beyond the target to ensure that the target intercepts most of the energy and ions flowing in the SOL. The choice of the boundary condition on the outermost SOL boundary should not influence the results. Plasma core is resolved radially into seven sub-regions by introducing flux surfaces based on vacuum field. For each radial region, density and 
temperature are prescribed according to the measured density and temperature profiles as shown in Fig 2. A peaked and a flat density profile, which are typical for low and high density plasmas under divertor operation [6,7], are chosen for investigation. The electron temperature profile is fixed during the computations since no significant change in $T_{\mathrm{e}}$-profile is observed experimentally between the low and high density plasmas [6,7]. The ion temperature profile, which determines the energy carried by the core CX-neutrals, has to be assumed to be as the same as the $\mathrm{T}_{\mathrm{e}}$-profile. Uncertainties in $\mathrm{T}_{\mathrm{i}}$-profile enter directly the calculation of the absolute iron sputtering yield, however, are not expected to significantly influence the parameter sensitivity studies presented in this paper. In the common SOL region, hydrogen plasma, carbon impurity and recycling neutrals are calculated self-consistently. Iron does not contribute to the charge and power balance. Hydrogen recycling coefficient on the target, baffle and wall is set to be unity. $2 \%$ (the ratio of the carbon-release to the hydrogen ion flux on the target) sputtering for carbon is assumed. The power entering the island $\mathrm{SOL}, \mathrm{P}_{\mathrm{SOL}}$, takes a value of $1.2 \mathrm{MW}$, which is equally spilt between ions and electrons and is fixed during the simulations. The separatrix density $\mathrm{n}_{\mathrm{es}}$ is varied from 1 to $5 \times 10^{19} \mathrm{~m}^{-3}$. Two sets of $\mathrm{D}$ (particle cross-field diffusivity) are used: 1) $\mathrm{D} \equiv 0.5 \mathrm{~m}^{2} / \mathrm{s}$ for all the $\mathrm{n}_{\mathrm{es}}$-points and 2) $\mathrm{D} \sim 1 / \mathrm{n}_{\mathrm{es}}$ for $\mathrm{n}_{\mathrm{es}} \leq 4 \times 10^{19} \mathrm{~m}^{-3}$ and $\mathrm{D}=0.5$ for $\mathrm{n}_{\mathrm{es}} \geq$ $4 \times 10^{19} \mathrm{~m}^{-3} \cdot \chi_{\mathrm{e}}$ and $\chi_{\mathrm{i}}$ are co-varied as $\chi_{\mathrm{e}}=\chi_{\mathrm{i}}=3 \mathrm{D}$.

\section{Simulation results}

\subsection{Energy spectrum and flux distribution of the neutrals on the wall}

As an example, Fig. 3 shows the energy spectrums of the neutrals to the wall resulting from two different input data sets: 1) $\mathrm{n}_{\mathrm{es}}=5 \times 10^{19} \mathrm{~m}^{-3}$ with $\mathrm{D}=0.5 \mathrm{~m}^{2} / \mathrm{s}$ and the flat $\mathrm{n}_{\mathrm{e}}$-profile and 2) $\mathrm{n}_{\mathrm{es}}=$ $1 \times 10^{19} \mathrm{~m}^{-3}$ with $\mathrm{D}=2 \mathrm{~m}^{2} / \mathrm{s}$ and the peaked $\mathrm{n}_{\mathrm{e}}$-profile. It is not yet clear whether $\mathrm{D}$ really increases with decreasing $\mathrm{n}_{\mathrm{es}}$ for the NBI-heated plasmas in W7-AS. The idea of the choice of an enhanced $D$ for the lower $n_{\text {es }}$-case in this example is to keep the product of $\chi_{i}$ and $n_{e s}$ and thereby the ion temperature in the islands roughly unchanged in the two cases in order to separate the effects of density from temperature. The impact of D on neutral flux and spectrum will be shown in next section. Calculated neutrals originating from the island SOL form a continuous spectrum because of the fine 3D spatial resolution for both plasma and neutrals. The spectrum curve of the higher- $\mathrm{n}_{\mathrm{es}}$ covers a larger area because of the enhanced recycling flux (see also next section). Furthermore, the spectrum is more peaked in the low energy range than that for the lower- $\mathrm{n}_{\mathrm{es}}$ case 
because of the shorter neutral penetration length in the islands. In contrast, the lower $n_{\mathrm{es}}$-case shows a much broader energy spectrum and the high-energetic particle flux even exceeds that in the enhanced recycling case, reflecting deeper penetration of the recycling neutrals in the islands. On the other hand, the calculated core CX-neutrals escaping from discrete radial zones cause discrete spectrums $\left(\mathrm{E}_{0}=3 / 2 \mathrm{~T}_{\mathrm{i}}\right)$ in the high energy range. This allows an easy identification of the origins of the core neutrals and a clear discrimination between the core and SOL neutrals as well. The two groups of the spectrum points differ not only in level but also in energy slope, which will be discussed below in some more detail.

Fig.4 shows the wall distributions of neutral flux in the high energy spectrum range with $\mathrm{E}_{0}>$ $180 \mathrm{eV}$ covering all the CX-neutrals escaping from the core. The two selected cases are compared with each other. The higher $\mathrm{n}_{\mathrm{es}}$ and the flat $\mathrm{n}_{\mathrm{e}}$-profile lead to an overall reduction of the 'high-energetic' neutral flux on the wall, which is already indicated by Fig. 3 and becomes clearer in Fig. 4. Note the difference in maximum between the two color tables. The 'highenergetic' neutrals are well kept outside the divertor chambers and concentrated in the toroidal range of the divertor modules. The strong reduction of the 'high-energetic' neutral outflow in the higher $\mathrm{n}_{\mathrm{es}}$ case results mainly from density effects and the in/out asymmetry in neutral flux there is due to the difference in island thickness between the inboard and outboard sides. In order to separate the neutral shielding effects of the edge islands from the core, $n_{\mathrm{e}}$-profile and $n_{\mathrm{es}}$ are varied independently, which gives rise to four possible combinations. The results are shown in Fig. 5, which shows the escaping possibilities of the CX-neutrals originating from different radial locations in the core, i.e. the possibility of a core CX-neutral particle reaching the wall. The escaping possibility decreases for the neutrals born in a deeper core region and the flat $\mathrm{n}_{\mathrm{e}}$-profile reduces the overall possibility to a lower level, especially for the neutrals coming from the plasma center. In contrast, the island density shows no influences on the deeply-located CXneutrals because the islands both in thickness and in density are much smaller than the core. However, unlike beam neutral particles, the recycling neutrals entering the core concentrate mainly at the edge region, especially in the flat density profile case. It is interesting to see from Fig. 5 that the four curves, which are grouped under the two core density profiles at the center, tend to regroup under the two island densities at the edge. This means that, in view of screening the recycling induced CX-neutrals, a dense island is more effective than a dense core. 


\section{2 Parameter sensitivities of the sputtering neutral flux}

Only the neutrals in the high energy spectrum range can produce iron impurity on the wall by means of physical sputtering and the sputtering yield is a sensitive function of the projectile energy and the incidence angle. The neutral flux integration weighted by the energy and incidence angle is done by the EIRENE code during the particle tracing, based on a sputtering model determined under laboratory conditions [8]. The sputtering yield calculated in this way provides an appropriate measuring quantity for assessing the divertor function on impurity production under different conditions. The sensitivities of the total iron yield to the input parameters listed in section 2 are summarized and compared in Fig. 6. Generally, the total iron yield decreases with increasing the island density and drops to a lower level if the density profile is switched from the peaked to the flat form. On the other hand, the choice of smaller cross-field transport coefficients leads only to slight increases of the iron yield even in the lowest $\mathrm{n}_{\mathrm{es}}$ case where $\mathrm{D}$ (and also $\chi_{\mathrm{e}}$ and $\chi_{\mathrm{i}}$ ) are varied within a factor of 4 . Reducing the cross-field transport increases the ion temperature in the islands (see Fig. 7) and extends the CX-neutral spectrum towards the high energy range. On the other hand, however, a reduced cross-field transport leads to a decrease of the total recycling flux (see Fig. 7). The two effects, to a certain extent, compensate each other.

For screening the sputtering CX-neutrals induced by the divertor recycling neutrals, the island density is the most effective parameter, while the core density and the profile form play a less important role within the high core density range investigated. The reason has been already partly given in the previous section. The baffles and the target form a divertor well for the recycling neutrals (see Fig. 1). Core plasma, as along as dense enough, works as a plug for the divertor well to prevent the most neutrals from escaping. In contrast, the edge islands are more transparent and cause the main leakage of the CX-neutrals. Furthermore, the surrounding islands form an intermediate layer between the core and the wall, moderating the interaction between the core plasma and the wall recycling neutrals. Obviously, the island density plays a key role in the both processes.

\section{Conclusion}

The transport of the wall-sputtering CX-neutrals induced by the divertor recycling neutrals in W7-AS is investigated using the EMC3/EIRENE code. A parameter sensitivity study is made in 
the parameter space of cross-field transport coefficients, core density profile and island density. The island density is scanned to cover different plasma confinement regimes. It is found that the island density is the most effective parameter for reducing the sputtering CX-neutral flux on the wall and thereby the iron production rate by different means, (a) by moving the CX-neutrals to low energy spectrum band, (b) by reducing the divertor-well leakage and (c) by moderating the interaction between the core plasma and the wall recycling neutrals. The core density and density profile show a strong effect on the neutrals from the core center region, however, a weak impact on the edge concentrated CX-neutrals resulting from the divertor recycling. The total iron production rate decreases roughly exponentially with increasing $n_{e s}$ and a flat $n_{e}$-profile further reduces the overall sputtering yield to a lower level. These results are not strongly affected by uncertainties in choosing the cross-field transport coefficients in the SOL since the resulting effects in ion temperature and total recycling flux tend to compensate each other.

\section{References}

[1] L. Giannone, R. Brakel et al., Plasma Physics and Controlled Fusion 45, 1713 (2003)

[2] Y. Feng, F. Sardei, P. Grigull et al., 32 ${ }^{\text {nd }}$ EPS, Tarragona (2005)

[3] R. Burhenn, J Baldzuhn, R. Brakel et al., Fusion Science Technol. 46(2004) 115-128

[4] Y. Feng, F. Sardei, J. kisslinger, J. Nucl. Mater. 266-269 (1999) 812.

[5] D. Reiter et al., “The EIRENE and B2-EIRENE codes”, Fus. Sci. Technol. 47 (2005) 172, and Ihttp:www.eirene.de

[6] K. McCormick, P. Grigull, R. Burhenn et al., Phys. Rev. Lett. 89, 015001 (2002)

[7] P. Grigull, K. McCormick, H. Renner et al., Fusion Eng. Design 66-68 (2003) 49-58

[8] W. Eckstein et al., , IPP-Report 9/82, Garching, Germany, 1993 


\section{Figure captions}

Fig. 1: Computation domain for EMC3/EIRENE simulation.

Fig. 2: Temperature and density profiles used in the simulations. The peaked and flat $\mathrm{n}_{\mathrm{e}-\mathrm{profile}}$ correspond to the typical low and high density divertor plasmas in W7-AS

Fig. 3: Examples of energy spectrum of the CX-neutrals hitting the wall for two cases with

1) $\mathrm{n}_{\mathrm{es}}=5 \times 10^{19} \mathrm{~m}^{-3}, \mathrm{D}=0.5 \mathrm{~m}^{2} / \mathrm{s}$, flat $\mathrm{n}_{\mathrm{e}}$-profle (solid curve and filled symbols)

2) $\mathrm{n}_{\mathrm{es}}=1 \times 10^{19} \mathrm{~m}^{-3}, \mathrm{D}=2 \mathrm{~m}^{2} / \mathrm{s}$, peaked $\mathrm{n}_{\mathrm{e}}$-profle.

SOL neutrals form continuous spectrums (curves), while those from the core introduce discrete points in the high energy range. The origins of the core neutrals can be identified from the energy spectrum. The different slopes guided by the two lines reflect different neutral penetration lengths resulting from the flat and peaked $\mathrm{n}_{\mathrm{e}}$-profles.

Fig. 4: Flux distributions of 'high-energetic' neutrals on the iron wall over a half field-period for the two cases as shown in Fig. 3. Left: the lower $n_{\text {es }}$ case. The front cut is an elliptical plane where the up and down divertor target and baffles are indicated by green colour. The neutral flux distribution on the graphite target and baffles is not shown.

Fig. 5: Island and core density effects on screening CX-nrutrals originating from different radial locations in the core.

Fig. 6: total iron production rate as a function of island density, cross-field transport coefficients and core density profile

Fig. 7: Total recycling flux and the separatrix ion temperature as a function of $\mathrm{n}_{\mathrm{es}}$ and cross-field transport coefficients 




Figure 1 


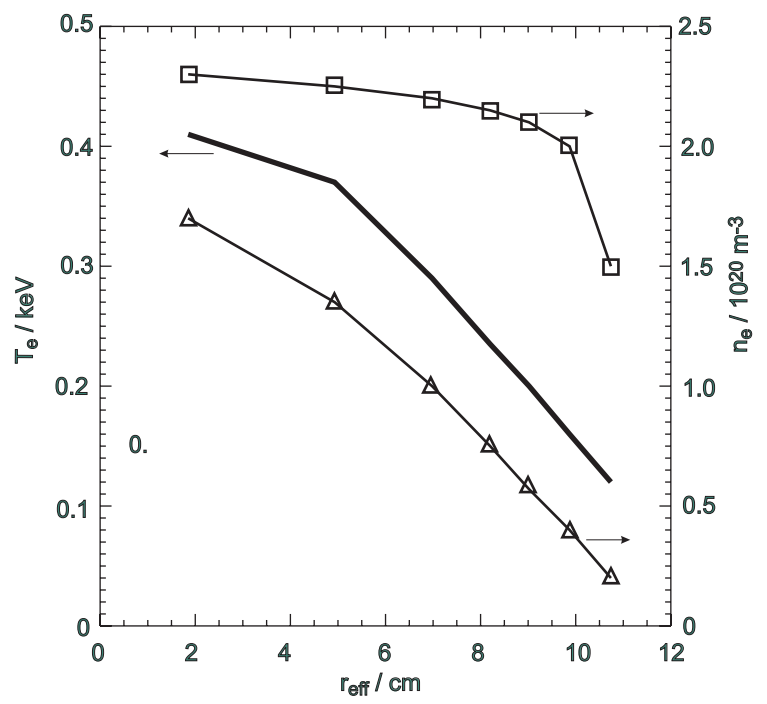

Figure 2 


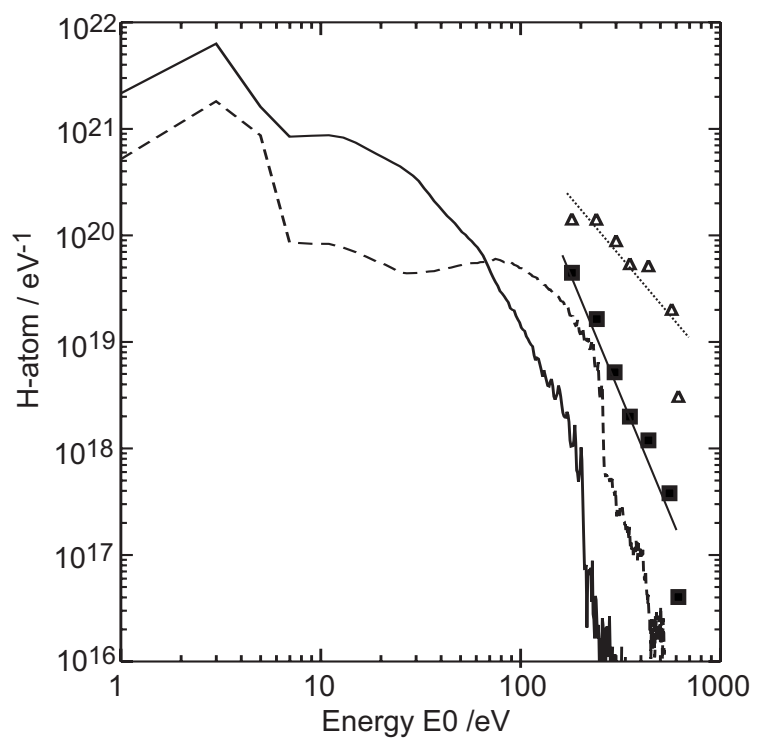

Figure 3 


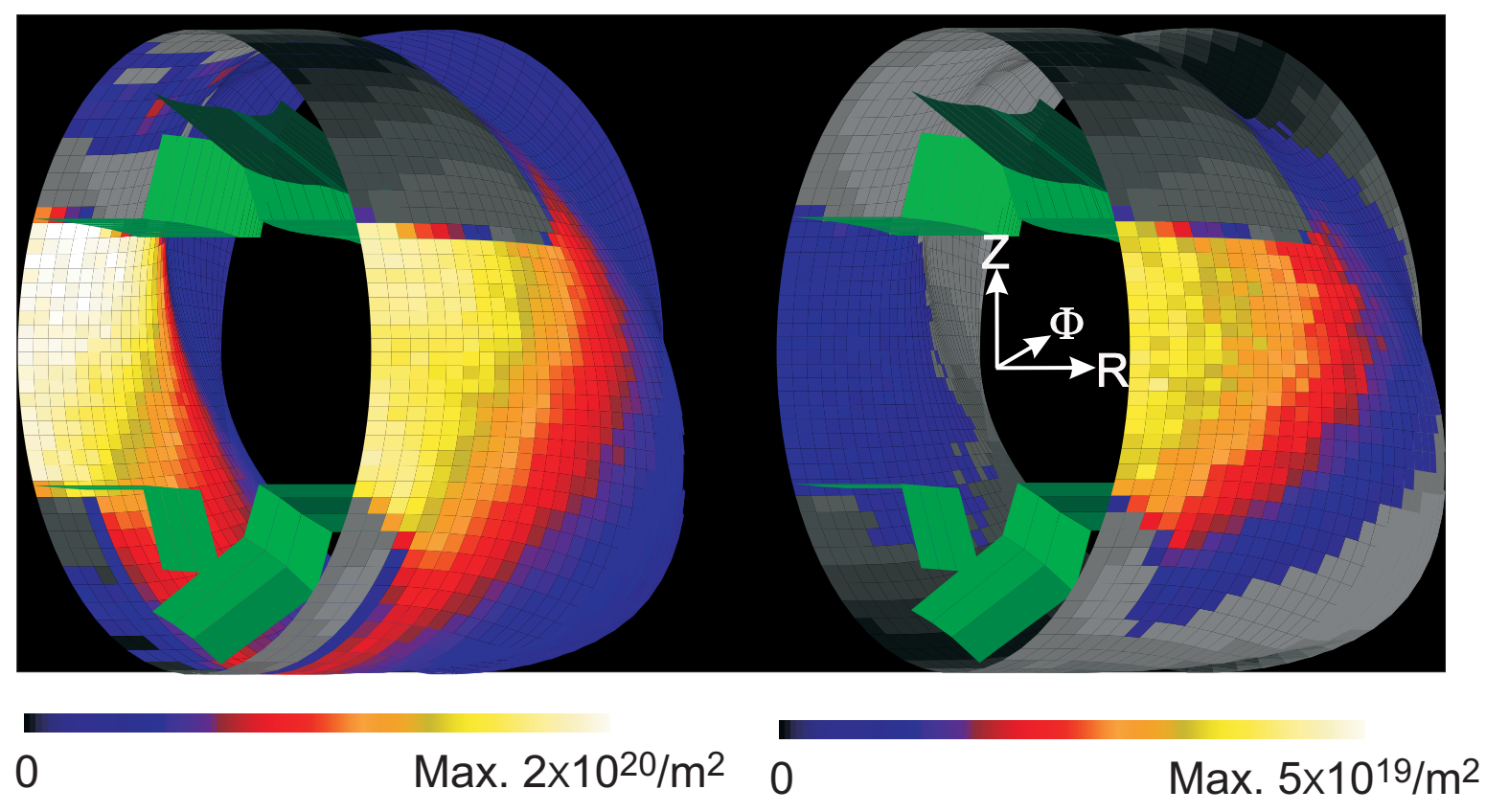

Figure 4 


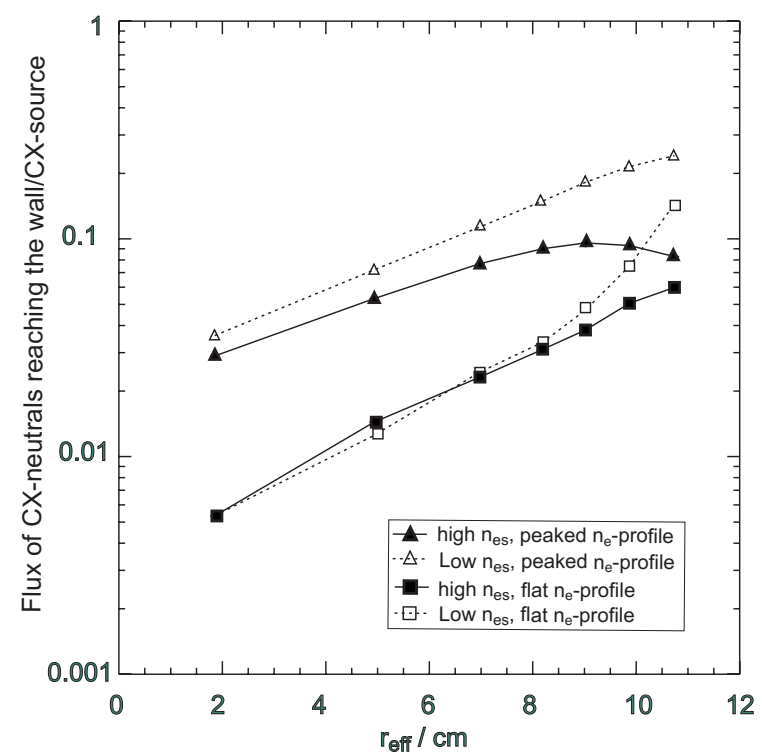

Figure 5 


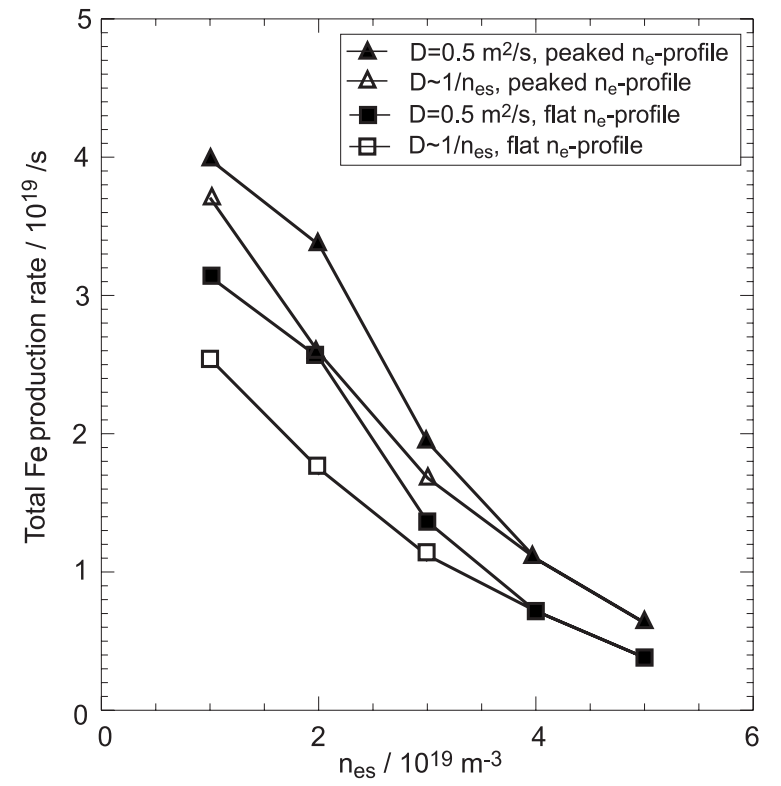

Figure 6 


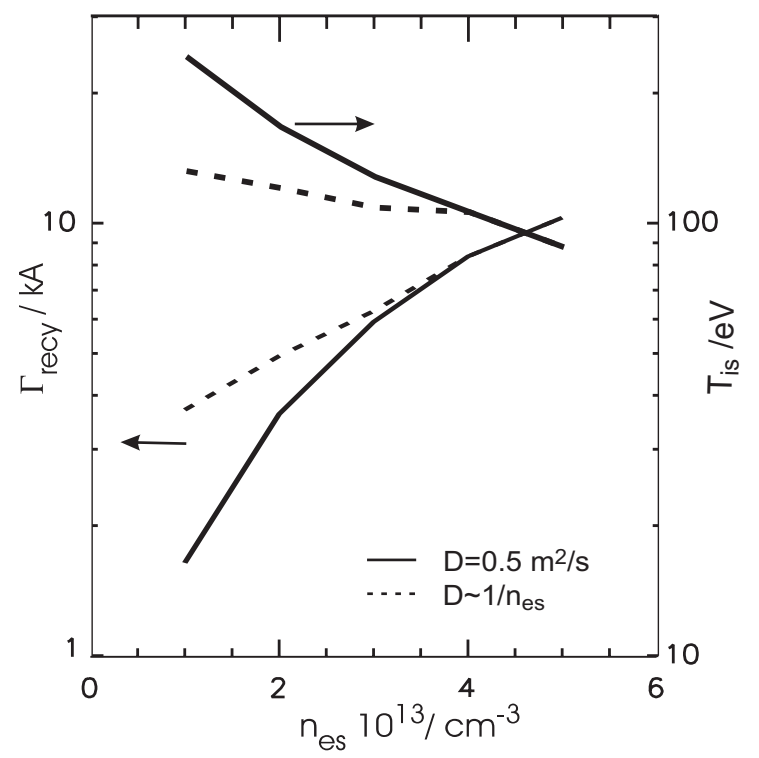

Figure 7 